ANNALES

POLONICI MATHEMATICI

LXXVII.1 (2001)

\title{
Second order differential inequalities in Banach spaces
}

\author{
by Gerd Herzog and Roland Lemmert (Karlsruhe)
}

\begin{abstract}
We derive monotonicity results for solutions of ordinary differential inequalities of second order in ordered normed spaces with respect to the boundary values. As a consequence, we get an existence theorem for the Dirichlet boundary value problem by means of a variant of Tarski's Fixed Point Theorem.
\end{abstract}

1. Introduction. Let $(E,\|\cdot\|)$ be a real normed space, ordered by a cone $K$ (i.e., a closed convex subset $K \neq \emptyset$ such that $\lambda K \subseteq K(\lambda \geq 0)$ and $K \cap(-K)=\{0\})$. A cone $K$ is called solid if its interior Int $K$ is not empty. The ordering is given by $x \leq y \Leftrightarrow y-x \in K$, and we write

$$
\begin{aligned}
x<y & \Leftrightarrow y-x \in K, x \neq y, \\
x \ll y & \Leftrightarrow y-x \in \operatorname{Int} K .
\end{aligned}
$$

Further notations are $E^{\star}$ for the topological dual of $E$ and

$$
K^{\star}=\left\{\varphi \mid \varphi \in E^{\star}, \varphi(x) \geq 0(x \in K)\right\} .
$$

If $K$ is solid, by the separation theorem of Hahn-Banach, for each $x_{0} \in \partial K$, the boundary of $K$, there exists a nontrivial $\varphi \in K^{\star}$ such that $\varphi\left(x_{0}\right)=0$; then $\varphi(x)>0$ for each $x \in \operatorname{Int} K$.

Consider a linear operator $A: E \rightarrow E, x \in K$ and a differentiable function $u:[0, T) \rightarrow E$. If $u(t) \geq 0(t \in(0, T])$ and

$$
\begin{aligned}
& u(0)=x, \\
& u^{\prime}(t) \leq A u(t), \quad 0 \leq t<T .
\end{aligned}
$$

then (cf. the proof of Theorem 1(A) in [8])

$$
x \in K, \varphi \in K^{\star}, \varphi(x)=0 \Rightarrow \varphi(A x) \geq 0 .
$$

On the other hand, if $K$ is solid and this condition holds for each $x \in K$, then $u^{\prime}(t) \geq A u(t)(t \in[0, T)), u(0) \geq 0$ imply $u(t) \geq 0$ for $t \in[0, T)([8]$, Theorem $1(\mathrm{~B}))$. In case Int $K=\emptyset$, additional assumptions on $E, K$ or $A$

2000 Mathematics Subject Classification: 34G20, 34B15, 34A40.

Key words and phrases: differential inequality, ordered normed space, Tarski's Fixed Point Theorem. 
are needed. In what follows we will investigate analogous implications for second order differential inequalities of the form

$$
\begin{aligned}
u^{\prime \prime}(t)+f(t, u(t)) & \geq v^{\prime \prime}(t)+f(t, v(t)) \quad(t \in(0,1)), \\
u(0) & \leq v(0), \\
u(1) & \leq v(1),
\end{aligned}
$$

the main assumption on $f$ being that it is quasimonotone increasing with respect to its second variable in the sense of Volkmann [9]:

$$
x, y \in D, x \leq y, \varphi \in K^{\star}, \varphi(x)=\varphi(y) \Rightarrow \varphi(f(t, x)) \leq \varphi(f(t, y)),
$$

together with a weak one-sided Lipschitz condition. A linear operator $A$ is called quasimonotone increasing if $x \mapsto A x$ has this property.

2. The linear case. For each $t \in(0,1)$ let $A(t): E \rightarrow E$ be linear. We say that $\mu:(0,1) \rightarrow \mathbb{R}$ has property $(\mathcal{P})$ if there exists a positive continuous function $\lambda:[0,1] \rightarrow \mathbb{R}$ such that

$$
\underline{D}^{2} \lambda(t):=\liminf _{h \rightarrow 0} \frac{\lambda(t+h)-2 \lambda(t)+\lambda(t-h)}{h^{2}}<-\mu(t) \lambda(t) \quad(t \in(0,1)) .
$$

For solutions of

$$
v^{\prime \prime}(t)+A(t) v(t) \leq 0,
$$

where

we have

$$
v^{\prime \prime}(t):=\lim _{h \rightarrow 0} \frac{v(t+h)-2 v(t)+v(t-h)}{h^{2}},
$$

THEOREM 1. Let $A(t): E \rightarrow E$ be quasimonotone increasing for each $t \in(0,1)$ and let there exist $p \in \operatorname{Int} K$ and a function $\mu$ with property $(\mathcal{P})$ such that $A(t) p \leq \mu(t) p(t \in(0,1))$. If $v:[0,1] \rightarrow E$ is continuous with $v(0) \geq 0, v(1) \geq 0$ and $v^{\prime \prime}(t)+A(t) v(t) \leq 0$ if $v(t) \notin K$, then $v(t) \geq 0$ $(t \in[0,1])$.

Proof. Choose a function $\lambda$ according to property $(\mathcal{P})$. Then there exists a minimal nonnegative $\varepsilon$ such that

$$
w(t)=v(t)+\varepsilon \lambda(t) p \in K \quad(t \in[0,1]) .
$$

If $\varepsilon>0$, then $w(0), w(1) \in \operatorname{Int} K$ and there is $t_{0} \in(0,1)$ such that $w\left(t_{0}\right) \in$ $\partial K$, so $v\left(t_{0}\right) \notin K$. Choose $0 \neq \varphi \in K^{\star}$ such that $\varphi\left(w\left(t_{0}\right)\right)=0$. Then $\varphi\left(A\left(t_{0}\right) w\left(t_{0}\right)\right) \geq 0$, and since $\varphi \circ w$ has a local minimum at $t_{0}$,

$$
\begin{aligned}
0 & \leq \underline{D}^{2}(\varphi \circ w)\left(t_{0}\right)=\varphi\left(v^{\prime \prime}\left(t_{0}\right)\right)+\varepsilon \underline{D}^{2} \lambda\left(t_{0}\right) \varphi(p) \\
& <-\varphi\left(A\left(t_{0}\right) v\left(t_{0}\right)\right)-\varepsilon \mu\left(t_{0}\right) \lambda\left(t_{0}\right) \varphi(p) \\
& =-\varphi\left(A\left(t_{0}\right) w\left(t_{0}\right)\right)+\varepsilon \lambda\left(t_{0}\right) \varphi\left(A\left(t_{0}\right) p\right)-\varepsilon \mu\left(t_{0}\right) \lambda\left(t_{0}\right) \varphi(p) \\
& \leq \varepsilon \lambda\left(t_{0}\right) \varphi\left(A\left(t_{0}\right) p-\mu\left(t_{0}\right) p\right) \leq 0,
\end{aligned}
$$

a contradiction. Therefore $\varepsilon=0$, which implies $v(t) \in K(t \in[0,1])$. 
Remarks. 1. The conditions of Theorem 1 imply uniqueness for

$$
x^{\prime \prime}(t)+A(t) x(t)=0 \quad(t \in(0,1)), \quad x(0)=x(1)=0 .
$$

2. In Theorem 1, neither $E$ is supposed to be complete nor $A(t)$ to be continuous.

3. In case $E=\mathbb{R}, K$ the set of all nonnegative reals, the conclusion of Theorem 1 remains valid if $v$ is merely continuous and if $v^{\prime \prime}$ is replaced by $\underline{D}^{2} v$; this will be used later.

4. A constant $\mu(t) \equiv \mu_{0}$ has property $(\mathcal{P})$ iff $\mu_{0}<\pi^{2}$.

3. The nonlinear case. To avoid the assumption Int $K \neq \emptyset$ we choose a different approach here. The function

$$
d(x)=\operatorname{dist}(x, K)
$$

is Lipschitz continuous with constant 1 and convex, and for $x \notin K$ we have

$$
d(x)=\sup \left\{-\varphi(x): \varphi \in K^{\star},\|\varphi\|=1\right\},
$$

since for $k \in K, \varphi \in K^{\star}$ such that $\|\varphi\|=1$ we have

$$
-\varphi(x) \leq \varphi(k-x) \leq\|k-x\|,
$$

from which the inequality " $\geq$ " readily follows; on the other hand, by the separation theorem applied to $K$ and the ball $B(x, d(x))$, there exists $\varphi \in K^{\star}$ with norm 1 such that (cf. [5])

$$
-\varphi(x)=\operatorname{dist}(x, \operatorname{ker} \varphi)=d(x),
$$

so the supremum in question is indeed a maximum.

If $w:[0,1] \rightarrow E$ has a second derivative at $t_{0} \in(0,1)$ and if $w\left(t_{0}\right) \notin K$, then for each $\varphi \in K^{\star}$ with $\|\varphi\|=1, d\left(w\left(t_{0}\right)\right)=-\varphi\left(w\left(t_{0}\right)\right)$ and each sufficiently small $h>0$ we get, for $\delta(t):=d(w(t))$,

$$
\frac{\delta\left(t_{0}+h\right)-2 \delta\left(t_{0}\right)+\delta\left(t_{0}-h\right)}{h^{2}} \geq-\varphi\left(\frac{w\left(t_{0}+h\right)-2 w\left(t_{0}\right)+w\left(t_{0}-h\right)}{h^{2}}\right),
$$

so

$$
\underline{D}^{2} \delta\left(t_{0}\right) \geq-\varphi\left(w^{\prime \prime}\left(t_{0}\right)\right) .
$$

From these considerations we deduce

TheOrem 2. Let $g:[0,1] \times E \rightarrow E$ satisfy

$$
\varphi(g(t, x)) \geq-\mu(t) d(x)
$$

if $t \in(0,1), x \notin K, \varphi \in K^{\star}, \varphi(x)=-d(x),\|\varphi\|=1$, where $\mu$ has property $(\mathcal{P})$. If $w:[0,1] \rightarrow E$ is continuous with $w(0) \geq 0, w(1) \geq 0$ and $w^{\prime \prime}(t)+g(t, w(t)) \leq 0$ if $w(t) \notin K$, then $w(t) \geq 0(t \in[0,1])$. 
Proof. For $\delta$ defined as above and $\varphi$ chosen such that $(\star)$ holds we get, in case $\delta(t)>0$,

$$
\underline{D}^{2} \delta(t) \geq-\varphi\left(w^{\prime \prime}(t)\right) \geq \varphi(g(t, w(t))) \geq-\mu(t) \delta(t),
$$

which (together with $\delta(0)=\delta(1)=0$ ) implies $\delta(t) \leq 0$, so $w(t) \in K$ $(t \in[0,1])$.

In order to give sufficient conditions for (7) to hold, consider

$$
t \in(0,1), x \in \partial K, \varphi \in K^{\star}, \varphi(x)=0 \Rightarrow \varphi(g(t, x)) \geq 0
$$

( $g$ is weakly inward with respect to $K$ ), and

$$
\begin{aligned}
& t \in(0,1), x_{0} \in \partial K, x \notin K, \\
& \begin{aligned}
\varphi \in K^{\star},\|\varphi\|=1, \varphi\left(x-x_{0}\right) & =-\left\|x-x_{0}\right\| \\
& \Rightarrow \varphi\left(g(t, x)-g\left(t, x_{0}\right)\right) \geq-\mu(t)\left\|x-x_{0}\right\|
\end{aligned}
\end{aligned}
$$

(this condition is a weakened one-sided Lipschitz condition).

LEMMA 1. Conditions (8) and (9) imply (7) under each of the following additional conditions:

(i) $K$ is a distance set.

(ii) $E$ is complete.

Proof. To prove (i), choose $x \notin K$ and $\varphi \in K^{\star}$ such that $\varphi(x)=-d(x)$, $\|\varphi\|=1$. Choose a nearest point $x_{0} \in \partial K$. Then $\varphi\left(x_{0}\right)=0$, so $\varphi\left(g\left(t, x_{0}\right)\right)$ $\geq 0$, and finally

$$
\varphi(g(t, x)) \geq \varphi\left(g(t, x)-g\left(t, x_{0}\right)\right) \geq-\mu(t)\left\|x-x_{0}\right\|,
$$

as asserted.

If $E$ is complete, by an adaptation of Lemma 2 in [7] to our situation, for $x \notin K, \varphi \in K^{\star}$ such that $\|\varphi\|=1, \varphi(x)=-d(x)$ and for each $\varepsilon>0$ there are $\varphi_{0} \in K^{\star}$ and $x_{0} \in \partial K$ such that

$$
\left\|\varphi_{0}\right\|=1, \quad\left\|\varphi_{0}-\varphi\right\| \leq \varepsilon, \quad \varphi_{0}\left(x_{0}\right)=0, \quad\left\|x-x_{0}\right\|<d(x)+\varepsilon .
$$

Then

$$
\begin{aligned}
\varphi(g(t, x)) & \geq \varphi_{0}(g(t, x))-\varepsilon\|g(t, x)\| \\
& \geq \varphi_{0}\left(g(t, x)-g\left(t, x_{0}\right)\right)-\varepsilon\|g(t, x)\| \\
& \geq-\mu(t)\left\|x-x_{0}\right\|-\varepsilon\|g(t, x)\| \\
& \geq-\mu(t) d(x)-\varepsilon|\mu(t)|-\varepsilon\|g(t, x)\|,
\end{aligned}
$$

and $\varepsilon \rightarrow 0$ proves the assertion.

REMARKS. 1. The need for additional conditions in Lemma 1 stems from the fact that in general a convex subset of an incomplete space need not have any supporting point (cf. [8]). 
2. If $K$ is normal and Int $K \neq \emptyset$, then the Minkowski functional of the order interval $[-p, p]$ ( $p$ a fixed interior point of $K$ ) is an equivalent norm, and with respect to this norm $K$ is a distance set: for $x \notin K, x+d(x) p$ is a nearest point in $K$. Then (see [4]) if $A(t)$ is quasimonotone increasing, then $A(t) p \leq \mu(t) p,\|\varphi\|=1, \varphi(x)=-\|x\|$ imply $\varphi(A(t) x) \geq-\mu(t)\|x\|$, so $(9)$ and a fortiori (7) hold.

In order to apply Theorem 2 to (3), (4) and (5), set

$$
g(t, x)=f(t, u(t)+x)-f(t, u(t)) \quad(t \in(0,1), x \in E)
$$

and

$$
w(t)=v(t)-u(t) \quad(t \in[0,1]) .
$$

Then $w(0) \geq 0, w(1) \geq 0, w^{\prime \prime}(t)+g(t, w(t)) \leq 0(t \in(0,1))$, and $g$ satisfies (8) if $f$ is quasimonotone increasing. If furthermore $f$ satisfies a one-sided Lipschitz condition (we do not insist on giving best possible conditions here) then

$$
\begin{array}{r}
t \in(0,1), x, x_{0} \in E, \varphi \in E^{\star},\|\varphi\|=1, \varphi\left(x-x_{0}\right)=-\left\|x-x_{0}\right\| \\
\quad \Rightarrow \varphi\left(f(t, x)-f\left(t, x_{0}\right)\right) \geq-\mu(t)\left\|x-x_{0}\right\|,
\end{array}
$$

then (9) holds for $g$. Therefore we have

TheOREM 3. Let $f:(0,1) \times E \rightarrow E$ be quasimonotone increasing and satisfy (10), where $\mu$ has property $(\mathcal{P})$. Then (3), (4) and (5) imply $u(t) \leq$ $v(t)(t \in[0,1])$ if $K$ is a distance set or $E$ is complete.

For later purposes we emphasize that (10) holds if $f$ satisfies the Lipschitz condition (L) of the next section.

4. An existence theorem. By means of the monotonocity results we are able to prove an existence theorem for the Dirichlet boundary value problem

$$
\begin{aligned}
y^{\prime \prime}(t)+f(t, y(t)) & =0 \quad(t \in(0,1)), \\
y(0)=y(1) & =0 .
\end{aligned}
$$

In case $E=\mathbb{R}^{n}$, a rather complete dicussion of (11), (12) may be found in Hartman [3], where a certain dependence of $f$ on $y^{\prime}(t)$ is allowed; here especially the theorems of Scorza Dragoni, Nagumo and Lettenmeyer should be mentioned. These theorems have been generalized to general Banach spaces by many authors (cf. [1]), where compactness conditions (or more general conditions involving measures of noncompactness) were involved, the main tool being Schauder-type fixed point theorems. Below, we will make use of a variant of Tarski's theorem.

Let us start with the case where $f$ satisfies a Lipschitz condition with respect to its second variable. We say that $\mu:[0,1] \rightarrow \mathbb{R}$ has property $\left(\mathcal{P}_{0}\right)$ 
if it is continuous and

$$
\lambda^{\prime \prime}(t)+\mu(t) \lambda(t) \leq 0
$$

has a positive solution in $C^{2}([0,1])$. Clearly, $\left(\mathcal{P}_{0}\right)$ implies $(\mathcal{P})$. Again, a constant $\mu(t) \equiv \mu_{0}$ has property $\left(\mathcal{P}_{0}\right)$ iff $\mu_{0}<\pi^{2}$, but there are nonconstant (even positive) functions $\mu$ having property $\left(\mathcal{P}_{0}\right)$ with arbitrarily large maximum (cf. [2], Chapter 4). Also, $\mu(t) \leq \pi^{2}, \mu \not \equiv \pi^{2}$ is sufficient for $\mu$ to have property $\left(\mathcal{P}_{0}\right)$, and finally a continuous $\mu$ has property $\left(\mathcal{P}_{0}\right)$ if $\lambda^{\prime \prime}(t)+\mu(t) \lambda(t)=0$ is disconjugate on [0,1] (cf. Hartman [3], Chapter XI, Corollary 6.1).

Lemma 2. Let $E$ be complete, $f:[0,1] \times E \rightarrow E$ be continuous and satisfy

$$
\|f(t, x)-f(t, \bar{x})\| \leq \mu(t)\|x-\bar{x}\| \quad(t \in[0,1], x, \bar{x} \in E),
$$

where $\mu$ has property $\left(\mathcal{P}_{0}\right)$. Then (11), (12) has a unique solution $y$.

If furthermore $\lambda$ is chosen according to property $\left(\mathcal{P}_{0}\right)$, then

$$
\|y(t)\| \leq C_{\lambda} \max _{t \in[0,1]}\|f(t, 0)\| \quad(t \in[0,1]),
$$

where the constant $C_{\lambda}$ depends only on $\lambda$, and

$$
\left\|y^{\prime}(t)\right\| \leq D_{\lambda, \mu} \max _{t \in[0,1]}\|f(t, 0)\| \quad(t \in[0,1]),
$$

where the constant $D_{\lambda, \mu}$ depends only on $\lambda$ and $\mu$.

Proof. We apply Banach's Fixed Point Theorem in $C([0,1], E)$ using the weighted maximum norm

$$
\|x\|_{\lambda}=\max _{t \in[0,1]} \frac{\|x(t)\|}{\lambda(t)}
$$

where $\lambda$ is chosen according to property $\left(\mathcal{P}_{0}\right)$. We rewrite (11), (12) as

$$
y(t)=\int_{0}^{1} G(t, s) f(s, y(s)) d s=: T(y)(t) \quad(t \in(0,1)),
$$

where

$$
G(t, s)= \begin{cases}s(1-t) & (0 \leq s \leq t \leq 1) \\ t(1-s) & (0 \leq t \leq s \leq 1)\end{cases}
$$

is Green's function. Then in $(0,1)$,

$$
\lambda(t)=\lambda(0)(1-t)+\lambda(1) t-\int_{0}^{1} G(t, s) \lambda^{\prime \prime}(s) d s,
$$


so for $x, \bar{x} \in C([0,1], E)$,

$$
\begin{aligned}
\|T(x)(t)-T(\bar{x})(t)\| & \leq \int_{0}^{1} G(t, s)\|f(s, x(s))-f(s, \bar{x}(s))\| d s \\
& \leq \int_{0}^{1} G(t, s) \mu(s) \lambda(s)\|x-\bar{x}\|_{\lambda} d s \\
& =\|x-\bar{x}\|_{\lambda}(\lambda(t)-(\lambda(0)(1-t)+\lambda(1) t)) .
\end{aligned}
$$

Therefore

$$
\|T(x)-T(\bar{x})\|_{\lambda} \leq q_{\lambda}\|x-\bar{x}\|_{\lambda}
$$

where

$$
q_{\lambda}=\max _{t}\left\{1-\frac{\lambda(0)(1-t)+\lambda(1) t}{\lambda(t)}\right\}<1
$$

so $T$ is a contraction with respect to the norm $\|\cdot\|_{\lambda}$ and thus has a unique fixed point $y$ which solves (11), (12). Finally,

$$
\|y\|_{\lambda} \leq \frac{\|T(0)\|_{\lambda}}{1-q_{\lambda}}
$$

and from this inequality appropriate constants $C_{\lambda}$ and $D_{\lambda, \mu}$ may easily be calculated.

The space $C([0,1], E)$ may be ordered by the cone

$$
K_{C}=\{x \mid x(t) \in K(t \in[0,1])\}
$$

set (with a fixed $l \geq 0$ )

$$
\begin{aligned}
\Lambda_{l}=\{x \mid x \in C([0,1], E), x(0) & =x(1)=0, \\
& \|x(t)-x(s)\| \leq l|t-s|(t, s \in[0,1])\} .
\end{aligned}
$$

In order to apply a variant of Tarski's Fixed Point Theorem we consider the following condition $(\mathrm{H})$ (see $[6]$ ) concerning the cone $K$ :

(H) each chain in $\Lambda_{l}$ has a supremum in $C([0,1], E)$.

(For a discussion of this property see Volkmann [10].)

The Fixed Point Theorem mentioned above reads as follows (see [6]):

Fixed Point Theorem. Let $M$ be a partially ordered set, $\Phi: M \rightarrow M$ increasing and such that each chain in $\Phi(M)$ has a supremum in $M$. If there is $v \in M$ with $v \leq \Phi(v)$ then $\Phi$ has a fixed point $x_{0}$ such that $v \leq x_{0}$.

We are now in a position to prove the following

Existence Theorem. Let $E$ be complete and let $K$ have property $(\mathrm{H})$. Suppose that

(i) there exists $v \in C^{2}([0,1], E)$ such that

$$
v^{\prime \prime}(t)+g(t, v(t))+h(t, v(t)) \geq 0 \quad(t \in[0,1]), \quad v(0) \leq 0, \quad v(1) \leq 0,
$$


(ii) $g:[0,1] \times E \rightarrow E$ is quasimonotone increasing and satisfies the conditions of Lemma 2 ,

(iii) $h:[0,1] \times E \rightarrow E$ is continuous, bounded and increasing with respect to its second variable on

$$
m_{v}=\{(t, x) \mid t \in[0,1], v(t) \leq x\} .
$$

Then problem (11), (12) with $f=g+h$ has a solution $y \geq v$.

Proof. We will apply the Fixed Point Theorem in

$$
M_{v}=C([0,1], E) \cap\{x \mid v(t) \leq x(t)(t \in[0,1])\},
$$

ordered by $K_{C}$. Choose $\lambda$ according to property $\left(\mathcal{P}_{0}\right)$ and define $\Phi: M_{v} \rightarrow$ $M_{v}$ by $y=\Phi(x)$, where $y$ is the solution of

$$
y^{\prime \prime}(t)+g(t, y(t))+h(t, x(t))=0, \quad y(0)=y(1)=0,
$$

by Lemma 2, where $f(t, \cdot)=g(t, \cdot)+h(t, x(t))$. Then if $\eta$ denotes a bound for $h$ on $m_{v}$, we have

$$
\left\|y^{\prime}(t)\right\| \leq D_{\lambda, \mu}\left(\max _{t \in[0,1]}\|g(t, 0)\|+\eta\right)=: l
$$

so

$$
\Phi(x) \in \Lambda_{l}
$$

for each $x \in M_{v}$ by the Mean Value Theorem. Furthermore, $\Phi$ is increasing since $x_{1} \leq x_{2}$ implies

$$
\begin{aligned}
\Phi\left(x_{2}\right)^{\prime \prime}(t)+g\left(t, \Phi\left(x_{2}\right)(t)\right) & +h\left(t, x_{1}(t)\right) \\
\leq & \Phi\left(x_{2}\right)^{\prime \prime}(t)+g\left(t, \Phi\left(x_{2}\right)(t)\right)+h\left(t, x_{2}(t)\right)=0 \\
= & \Phi\left(x_{1}\right)^{\prime \prime}(t)+g\left(t, \Phi\left(x_{1}\right)(t)\right)+h\left(t, x_{1}(t)\right),
\end{aligned}
$$

so $\Phi\left(x_{1}\right) \leq \Phi\left(x_{2}\right)$ according to Theorem 3 , applied to $f(t, \cdot)=g(t, \cdot)+$ $h\left(t, x_{1}(t)\right)$. Finally, by a similar reasoning, $\Phi(v) \geq v$, so the Fixed Point Theorem applies.

REMARKS. 1. The following example may be considered in various spaces of real sequences: Let $g=0$ and $h=\left(h_{n}\right)_{n \in \mathbb{N}}$ be defined by

$$
h_{n}(t, x)=\pi^{2} \min \left\{\max \left\{0, x_{n}\right\}, 1\right\}+1 / n \quad\left(t \in[0,1], x=\left(x_{1}, x_{2}, \ldots\right)\right) .
$$

Then $h$ is continuous, bounded and increasing in $c_{0}$ (the space of all zero sequences), in $c$ (the space of all convergent sequences), and in $l^{\infty}(\mathbb{N})$, all equipped with the supremum norm and the natural cone. Then $g+h$ satisfies the conditions of the Existence Theorem (in (iii) one may choose $v=0$ ), and $(\mathrm{H})$ holds in $l^{\infty}(\mathbb{N})$ but neither in $c$ nor in $c_{0}$; in the latter case, (11), $(12)$ has no solution, whereas in $l^{\infty}(\mathbb{N})$ there are infinitely many solutions. Of course, this example might be modified in various ways, e.g., $g$ may be chosen to satisfy (i), $\pi^{2}$ may be replaced by an arbitrary nonnegative constant and $(1 / n)_{n \in \mathbb{N}}$ by a suitable sequence. 
2. Condition (iii) holds if: There exists $p \in K$ such that

$$
h(t, x) \geq p \quad(t \in[0,1], x \in E) .
$$

Then $v$ may be chosen as the solution of $v^{\prime \prime}(t)+g(t, v(t))+p=0, v(0)=$ $v(1)=0$. Such a $p$ exists if $\operatorname{Int} K \neq \emptyset$.

3. If there is also $w \in C^{2}([0,1], E)$ such that

$$
w^{\prime \prime}(t)+g(t, w(t))+h(t, w(t)) \leq 0 \quad(t \in[0,1]), \quad w(0) \geq 0, \quad w(1) \geq 0
$$

and $v \leq w$, then condition (iii) is only needed on

$$
m_{v, w}=\{(t, x) \mid t \in[0,1], v(t) \leq x \leq w(t)\} ;
$$

the Fixed Point Theorem may then be applied with

$$
M_{v, w}=C([0,1], E) \cap\{x \mid v(t) \leq x(t) \leq w(t)(t \in[0,1])\}
$$

and gives a solution $y$ such that $v \leq y \leq w$.

5. An example. Let $E=l^{\infty}(\mathbb{Z} \times \mathbb{Z})$ equipped with the supremum norm and let $K$ denote the natural cone, which has property $(\mathrm{H})$. Let $\Delta: E \rightarrow E$ be defined by

$$
(\Delta x)_{i, j}=x_{i+1, j}+x_{i-1, j}+x_{i, j+1}+x_{i, j-1}-4 x_{i, j}, \quad x=\left(x_{i, j}\right) \quad(i, j \in \mathbb{Z}),
$$

and by means of an increasing, continuous, bounded function $h_{0}: \mathbb{R} \rightarrow \mathbb{R}$ set

$$
(h(x))_{i, j}=h_{0}\left(x_{i, j}\right) \quad(i, j \in \mathbb{Z}) .
$$

$\Delta$ is quasimonotone increasing since it is the sum of an increasing function and a scalar multiple of the identity, and Lipschitz continuous with constant 8; $h: E \rightarrow E$ is continuous and monotone increasing. Consider

$$
y^{\prime \prime}(t)+\mu(t) \Delta y(t)+h(y(t))=r(t) \quad(t \in[0,1]), \quad y(0)=y(1)=0,
$$

where $\mu \geq 0,8 \mu$ has property $\left(\mathcal{P}_{0}\right)$ and $r:[0,1] \rightarrow E$ is continuous. Choose $r_{0}$ and $r^{0}$ such that

$$
r_{0} \leq r_{i, j}(t) \leq r^{0} \quad(i, j \in \mathbb{Z}, t \in[0,1]),
$$

and functions $v_{0}, w_{0} \in C^{2}([0,1], \mathbb{R})$ such that $v_{0} \leq w_{0}$,

$$
\begin{gathered}
v_{0}(0) \leq 0 \leq w_{0}(0), \quad v_{0}(1) \leq 0 \leq w_{0}(1) \\
v_{0}^{\prime \prime}(t)+h_{0}\left(v_{0}(t)\right)-r^{0} \geq 0 \geq w_{0}^{\prime \prime}+h_{0}\left(w_{0}(t)\right)-r_{0} \quad(t \in[0,1])
\end{gathered}
$$

(this may be done in many ways), and set $v_{i, j}(t)=v_{0}(t), w_{i, j}(t)=w_{0}(t)$. Then the Existence Theorem (together with Remark 3 following it) is applicable and gives a solution of the above boundary value problem. 


\section{References}

[1] S. R. Bernfeld and V. Lakshmikantham, Linear monotone method for nonlinear boundary value problems in Banach spaces, Rocky Mountain J. Math. 12 (1982), 807-815.

[2] P. Hannig, Das Schießverfahren zur Gewinnung von Existenz- und Eindeutigkeitsaussagen bei Sturm-Liouville-Randwertaufgaben, Dissertation, Karlsruhe, 1996.

[3] P. Hartman, Ordinary Differential Equations, Wiley, New York, 1964.

[4] G. Herzog, One-sided estimates for linear quasimonotone increasing operators, Numer. Funct. Anal. Optim. 19 (1998), 549-555.

[5] R. Lemmert, Über die Invarianz konvexer Teilmengen eines normierten Raumes in bezug auf elliptische Differentialgleichungen, Comm. Partial Differential Equations 3 (1978), 297-318.

[6] —, Existenzsätze für gewöhnliche Diferentialgleichungen in geordneten Banachräumen, Funkcial. Ekvac. 32 (1989), 243-249.

[7] R. Lemmert und P. Volkmann, Randwertprobleme für gewöhnliche Differentialgleichungen in konvexen Teilmengen eines Banachraumes, J. Differential Equations 27 (1978), 138-143.

[8] - - - On the positivity of semigroups of operators, Comment. Math. Univ. Carolin. 39 (1998), 483-489.

[9] P. Volkmann, Gewöhnliche Differentialungleichungen mit quasimonoton wachsenden Funktionen in topologischen Vektorräumen, Math. Z. 127 (1972), 157-164.

[10] —, Über Kegel, welche einer Bedingung von Lemmert genügen, Seminar LV, No. 7 (2000) (http://www.mathematik.uni-karlsruhe.de $/{ }^{\sim}$ semlv).

Math. Institut I

Universität Karlsruhe

Englerstr. 2

76128 Karlsruhe, Germany

E-mail: gerd.herzog@math.uni-karlsruhe.de roland.lemmert@math.uni-karlsruhe.de 